\title{
Psychological aspects of ortho-surgical protocols
}

\author{
A. Clément ${ }^{1}$, H. Guilyardi ${ }^{2}$ M. Makaremi ${ }^{3}$, P. Goudot $^{4}$, T. Schouman $^{5}$ \\ 1 Clinical Psychologist, Unit of Psychology, Psychotherapy, Psychoanalysis and Psychiatry. \\ Maxillofacial Surgery Services, Pr. P Goudot, Pitié-Salpêtrière Hospital \\ 2 Psychiatrist, Unit of Psychology, Psychotherapy, Psychoanalysis and Psychiatry \\ Maxillofacial Surgery Services Pr. P Goudot, Pitié-Salpêtrière Hospital, and Clinical Research Unit \\ Pr. A Mallet, Mazarin Division, Pitié-Salpêtrière Hospital \\ ${ }^{3}$ Certified Specialist in Dentofacial Orthopaedics, private practice \\ ${ }^{4}$ Maxillofacial surgeon, University Professor and head of the Stomatology and Maxillofacial \\ Services at Pitié-Salpêtrière Hospital \\ ${ }_{5}$ Maxillofacial surgeon, Hospital practitioner, Maxillofacial Surgery Services Pr. P Goudot, \\ Pitié-Salpêtrière Hospital
}

\begin{abstract}
By the results of an exploratory research, we were able to put forward the specificity of the request in ortho-surgical protocol. Even if many studies evaluated correlations between psychological profiles and postoperative dissatisfaction, no one asked for the link between initial request and postoperative dissatisfaction. We suggest setting up an observation and forwardlooking research's protocol: 100 patients must been followed in a longitudinal way during 3 years (medium time between the first consultation and 6 months after the surgery). The whole duration of the study is about 4 years. It's divided in three times: treatment's proposal, preoperative hospitalization's day, postoperative hospitalization's day (three psychological interviews after the auto-administration of a clinical questionnaire, the Rathus Scale, the Hamilton depression and anxiety scale and the SF-36 quality of life scale. The research's main objective is to study the correlation between initial request and the satisfaction level. The second objectives are the evaluation of self-respect, anxiety, depression, and quality of life scales at the three times of the research, and the evaluation of the psychological following's impact on the results. Thirdly, we want to set up a questionnaire upon the initial request to help the practitioners.
\end{abstract}

\section{KEYWORDS}

Ortho-surgery, treatment's request, quality of life, dissatisfaction, self-respect, psychological risk, depression

\section{INTRODUCTION}

One of the characteristics of requests to undergo orthognathic surgical interventions as compared with other maxillofacial surgical interventions is the nonvital aspect of such surgeries. The interdisciplinary exchanges that have emerged from our collaboration with psychologists and psychoanalysts

Address for correspondence:

Adèle Clément

39 rue de Lisbonne

75008 Paris

E-mail: abdl7@hotmail.fr

Article received: 30-09-2015. Accepted for publication: 25-10-2015.

This is an Open Access article distributed under the terms of the Creative Commons Attribution License (http://creativecommons.org/licenses/by/4.0), which permits unrestricted use, distribution, and reproduction in any medium, provided the original work is properly cited. 
from Dr. H Guilyardi's Unit of Psychotherapy and the orthognathic surgical team of Professor P Goudot's Maxillofacial Surgery Services, as well as the implementation of an expanded support system for patients, have led us to enlist the services of psychologists in orthognathic surgical protocols. The major difficulties encountered independent of the success of the medical treatment are:

- Interruptions during treatment;

- Concerns expressed by patients regarding the sensitive and esthetic modifications involved;

- Anxiety before surgery, causing delays or repeated absences, posttreatment dissatisfaction sometimes leading to requests for repeat surgery, conflicts with the care team, injunctions filed for reparation of losses experienced, and even to judicial procedures.

- The a priori decision to pursue surgical treatment is primarily dependent on the patients' choice. This choice is determined by functional, esthetic, and preventive criteria, which are discussed during orthodontic treatment and at the time of medical and surgical consultations. However, this choice causes tensions between expectations and potential modifications or even losses.

From as early as the first meeting, the patients come with a request that will then be organized according to treatment proposals. Depending on the case, the request may match the proposals; sometimes, there may be a mismatch between the patients' request and the proposed treatment, i.e., sometimes the envisaged procedure exceeds patient expectations while at other times these expectations go beyond the limits of the surgical procedure. In all eventualities, this request is structured around three questions: Who is talking? What are they talking about? Who are they talking to?

Who is talking? These expectations are based on everyday life and take on a different importance to each person: self-perception, interpersonal relationships, one's role in the professional world, friendliness, family-orientedness, desire for acknowledgment, difficulties with social inclusion, feelings of inferiority.

What is the speaker talking about? The patients come with certain expectations that can be grouped into three categories: esthetic, functional, and preventive. Esthetic expectations include having aligned teeth, a more beautiful smile, and less visible gums. Functional expectations are being able to eat better and having improved elocution, breathing, mouth closure; pain relief; and amelioration of sleep apnea. Finally, preventive expectations include avoiding tooth loosening, overlap, and accentuated discrepancies.

To whom is the speaker talking? The doctors, who represent the voice of knowledgeable, are being addressed. Their response therefore has the potential to modify the request either by reinforcing (for example adding an esthetic aspect to a functional demand), shifting the perspective (the problem may not just affect tooth alignment but also the position of the jaws), or decreasing the request (such as sleep apnea that is not necessarily treated with ortho-surgery. This manner of address implies an intention behind the request. 


\section{THE STATE OF THE QUESTION}

According to studies, 52\%-74\% patients consider the esthetic aspect to be the deciding factor when requesting treatment ${ }^{10}$. Requests for orthosurgery and requests for esthetic surgery are based on similar psychological motivations ${ }^{11}$. On an average, the rate of satisfaction is very high at $90 \%$, with only $10 \%$ patients claiming dissatisfaction ${ }^{6,9}$. The reasons given for dissatisfaction appear more linked to psychological elements rather than the failure of the medical procedure ${ }^{19}$.

The psychological elements put forward to justify this dissatisfaction include problems such as body dysmorphic disorder ${ }^{21}$, depression ${ }^{16}$, anxiety $^{4}$, and neuroticism ${ }^{1}$. A recent study ${ }^{7}$ has shown the presence of problems of body dysmorphic disorder, anxiety, and depression among patients who request orthognathic surgical treatment. The correlation between these psychopathological elements and posttreatment dissatisfaction has also been demonstrated ${ }^{12,13}$. Depression has been put forward as a major cause of dissatisfaction and its intensity does not vary despite the success of treatment ${ }^{8}$.

However, to the extent of our knowledge, none of the studies that focus on the psychological aspects ${ }^{2}$ have considered the psychological mechanisms involved in patients' requests for treatment, or the psychological tensions associated with the experience of having such a deformity as described by patients during their care. These studies have basically been done to distinguish psychological profiles ${ }^{17}$ and to determine the psychopathological elements associated with dissatisfaction ${ }^{5,18}$. They do not offer any information on the specificity of these requests for treatment nor the relationship between the initial request and final satisfaction.

\section{PILOT STUDY}

\section{Materials}

We have highlighted these psychological conflicts and dynamics in a prospective study involving the weekly psychological follow-up of 22 patients (eight postoperative, nine post-initial consultation, and five preoperative patients) over the course of a year. We also include routine interviews with 20 patients during their initial consultation, 110 preoperative visit patients ( 1 month before surgery), and 58 postoperative visit patients (1 year after surgery; 6 months after the end of treatment). The secondary objective of our study was to identify the warning signs for feelings of being overwhelmed that are expressed by patients and could pose major psychological risks. 


\section{Methods}

An exploratory study was conducted from September 2012 to June 2014, centering on the participation of psychologists in multidisciplinary consultations, the psychological follow-up of patients after these consultations, and the routine clinical questionnaire survey results obtained in Dr. Guilyardi's Unit of Psychotherapy during both preoperative and postoperative day visit (GNDG quantitative questionnaire; Goudot $P$, Ney M, Demeule C, Guilyardi H).

The collected data are based on these questionnaires and on qualitative analyses of discourses. The hypotheses were that constants exist in psychological adjustments associated with the phases of a protocol and that gaps exist between these constants associated with an overflow of capacities for redevelopment and indicating a psychological risk. On the basis of these hypotheses, we wish to highlight the appearance of particular elements in patients' discourses that indicate the need for psychological care during the different stages of treatment.

\section{Results}

\section{Quantitative data from 110 questionnaires (Figure 1)}

It is interesting to note that this surgery is essentially tailored to a young population but can also be recommended to patients aged $\geq 60$ years. The majority of requests (90\%) were made after a medical consultation, but the majority of the patients (59\%) believed that they were the ones to initiate the process. This reflects patients' investment in a process that is not merely limited to the medical procedure itself and indicates elevated patients' motivation (2.3/3). The reason provided quasi-systematically (94\%) is functional, although the results indicate that on average, only $38 \%$ patients experience functional discomfort. The observations noted while conducting the interviews with the patients allow us to shed light on this apparent contradiction; several patients cited as their motivation the prevention of potential problems in the future (tooth extraction, loosening of teeth, difficulties chewing) during their medical consultation.

Although only half the patients felt sure of themselves and only a small majority felt they got on well with others, 86\% patients questioned whether they were well-accepted by others. This shows the gap between social self-perception and social acceptance (Figure 2). Once again, the clinic has permitted us to explore this gap; because of a lack of self-confidence brought on by deformity, several patients feel restricted in their emotional and/or professional roles.

"I can't smile," "I remain as inconspicuous as possible," "I would like to be more personable but I dare not because of my teeth." Nonetheless, patients admitted that their friends and family did not seem to notice their deformity. Moreover, the rates of satisfaction with social and family life appeared slightly more elevated than satisfaction with professional, school, and emotional life. 


\begin{tabular}{|c|c|c|}
\hline General Profile & $\begin{array}{r}\text { No } \\
\text { Mea } \\
\text { Reaso } \\
\text { Motivation: average }\end{array}$ & $\begin{array}{l}\text { valence for sex and socioprofessional situation } \\
\text { e: } 28 \text { years (range: } 14-63 \text { years; mode: } 16 \text { years) } \\
\text { ven for intervention: } 94 \% \text { functional, } 45 \% \text { esthetic } \\
3 \text { mode et median: } 2 \text {. Scoring on a scale of 1-3: (1) unmotivated, } \\
\text { (2) motivated, (3) very motivated. }\end{array}$ \\
\hline $\begin{array}{l}\text { Overall } \\
\text { profile }\end{array}$ & $\begin{array}{r}\text { No pre } \\
\mathrm{M} \in \\
\text { Reason g } \\
\text { Motivation: Mean }\end{array}$ & $\begin{array}{l}\text { nce according to sex or socioprofessional situation } \\
\text { age of } 28 \text { (range: } 14-63 \text { years; mode: } 16 \text { years) } \\
\text { for treatment: functional in } 94 \% \text { and esthetic in } 45 \% \\
2.3 / 3 \text {, mode and median of } 2 \text {. Rated on a scale of } 1-3: \text { (1) Less } \\
\text { tivated, (2) motivated, (3) very motivated. }\end{array}$ \\
\hline $\begin{array}{c}\text { Treatment } \\
\text { process }\end{array}$ & $\begin{array}{r}\text { For } 4 \\
\text { The average age for col }\end{array}$ & $\begin{array}{l}\text { patients were referred by their orthodontist } \\
10 \% \text { by their attending physician } \\
\% \text { by another person (generally a dentist) } \\
\text { this process is started through personal initiative } \\
\text { encing treatment is } 21.5 \text { years (range: } 7-51 \text { years, mode: } 14 \text { years) }\end{array}$ \\
\hline \multirow{3}{*}{$\begin{array}{c}\text { Self- } \\
\text { perception }\end{array}$} & Physical & $\begin{array}{c}\text { Harmonious face: } 32 \% \\
\text { Face representing a hindrance: } 42 \% \\
\text { Displeasing mirror reflection: } 27 \% \\
\text { Fear of looks from others: } 33 \% \\
\text { Sense of suffering related to disharmony: } 33 \% \\
\text { Positive self-perception on a physical level: Average of } 60 \%\end{array}$ \\
\hline & Psychological & $\begin{array}{c}\text { Appearance matches personality: } 33 \% \\
\text { Self-assuredness: } 33 \% \\
\text { Gets on easily with others: } 60 \% \\
\text { Well accepted by others: } 86 \% \\
\text { Positive self-perception on a psychological level: Average of } 62 \%\end{array}$ \\
\hline & Overall self-esteem: $76 \%$ & $\begin{array}{c}\text { Satisfactory social life: } 78 \% \\
\text { Satisfying professional life: } 69 \% \\
\text { Satisfying family life: } 86 \% \\
\text { Satisfying emotional life: } 72 \%\end{array}$ \\
\hline \multirow{3}{*}{ Health } & General & $\begin{array}{l}\text { Following medical treatment: } 33 \% \\
\text { Previous hospitalization: } 66 \% \\
\text { Previous surgical intervention: } 55 \% \\
\text { Preoccupation with health: } 59 \%\end{array}$ \\
\hline & Physical & $\begin{array}{l}\text { Average functional discomfort: } 38 \% \\
\text { Breathing discomfort: } 33 \% \\
\text { Mastication discomfort: } 46 \% \\
\text { Elocution discomfort: } 55 \% \\
\text { Swallowing discomfort: } 23 \% \\
\text { Pains: } 37 \%\end{array}$ \\
\hline & Psychological & $\begin{array}{l}\text { Good average psychological health: } 63 \% \\
\text { Feeling depressed: } 19 \% \\
\text { Feeling anxious: } 26 \% \\
\text { Perceives themselves as sentimental: } 52 \% \\
\text { Fatigue: } 40 \% \\
\text { Sleep disturbance: } 28 \% \\
\text { Previous psychological monitoring: } 37 \%\end{array}$ \\
\hline
\end{tabular}

Figure 1

Quantitative preoperative data obtained from 110 questionnaires. 


\section{Qualitative data}

The qualitative data (Figure 3) collected from the multidisciplinary consultations suggest that four stages exist in orthognathic surgical protocols. These four stages dictate the amount of time needed for individual psychological development. We have identified the prevalent psychological adjustments that occur during these various stages and the major difficulties that are encountered by some patients, and consequently require another consultation or psychological follow-up.

Stage 1: Initial request and treatment proposal

The patients and their loved ones (especially if they are minors) must take time to reflect upon their initial request. If this request is primarily functional, the patients' involvement will be measured by assessing their level of daily discomfort and how treatment will contribute to dealing with difficulties related to this discomfort. This evaluation can only be performed by the patients themselves once the expected benefits of treatment and the risks of a new form of discomfort (primarily loss of sensation in the lower lip) have been explained. The prospect of surgery can also appear frightening; surgical intervention is an invasive act that not only has inherent risks but also involves hospitalization and a time of remission.

The initial disharmony that the patients wish to correct takes the form of a sickness that must be cured, which modifies the image that they have of themselves and causes them to become ill. We also recorded statements such as: "Now that I know what I have, I cannot stay like this; I feel obligated to undergo treatment," or "I have always known that there was something wrong; that is what has prevented me from approaching others and from having self-confidence." Two risks now emerge: the perception of a medical obligation (or urgency) and a causal attribution of disharmony to an organic pathology responsible for the patients' personal failures.

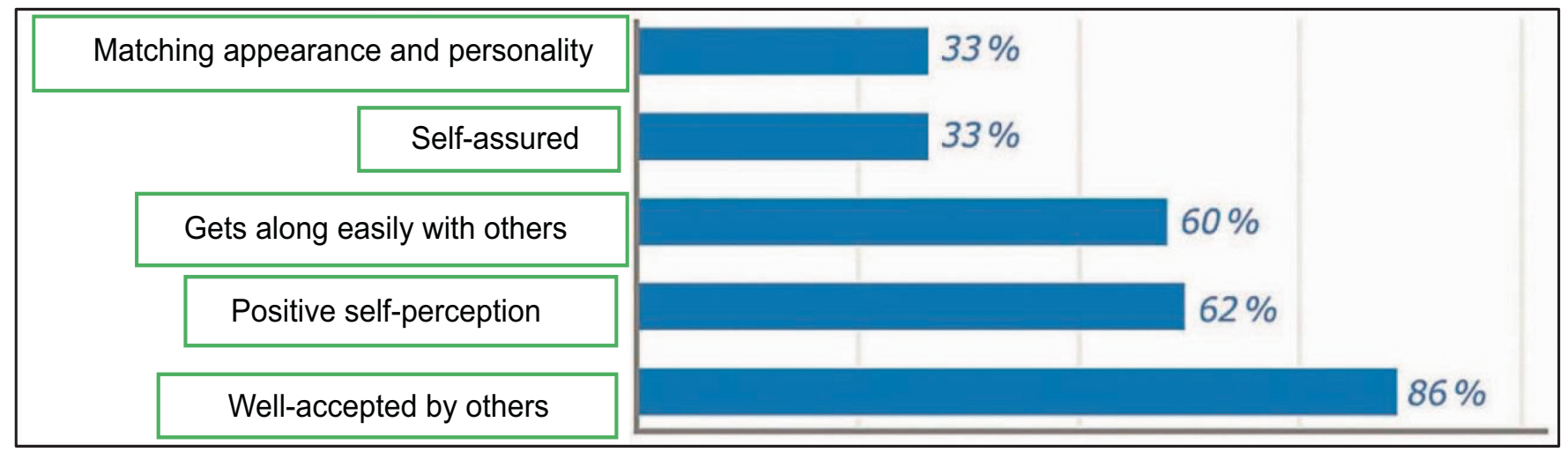

Figure 2

Psychological perception of oneself and others at the preoperative stage. 

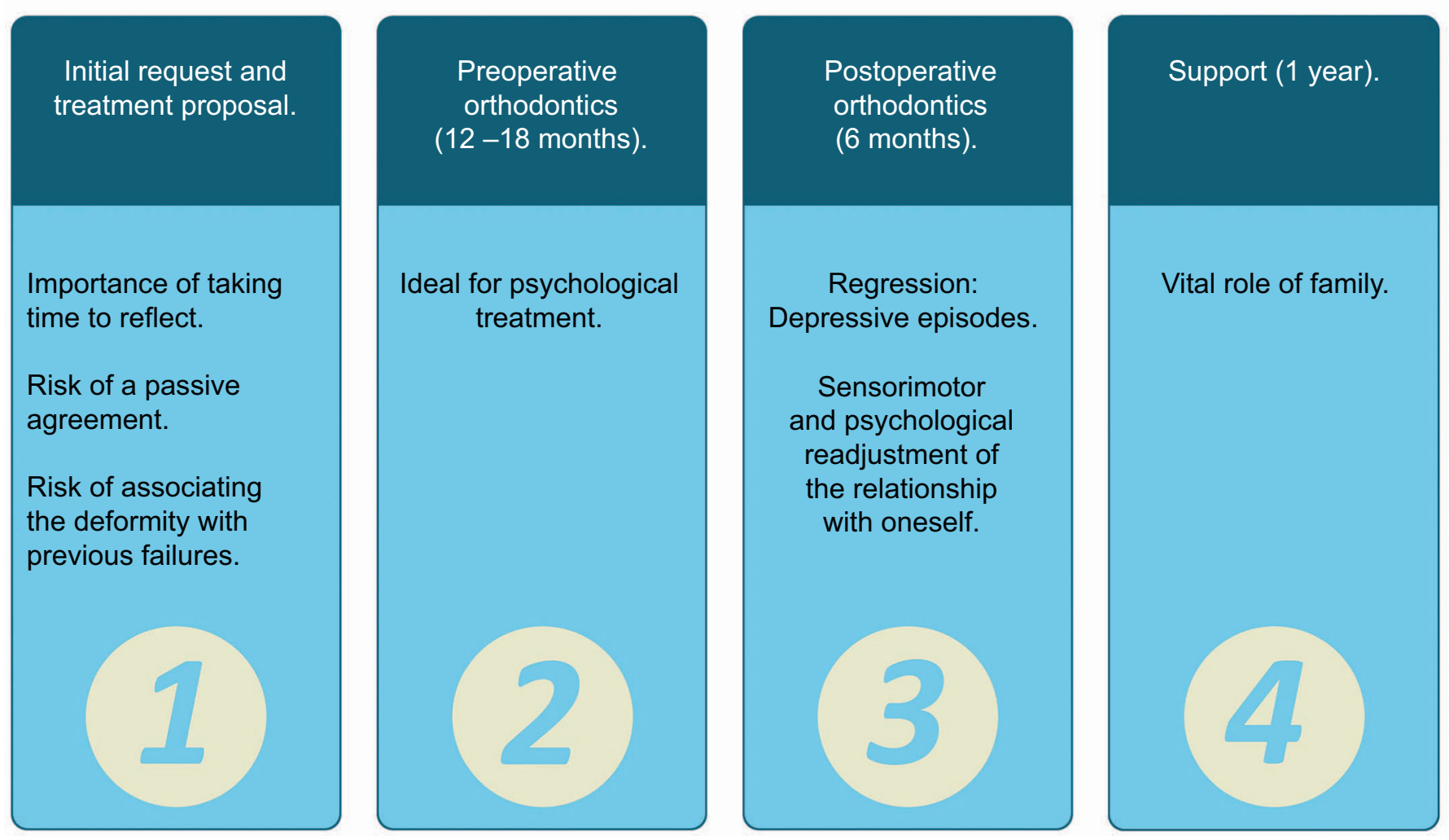

Figure 3

Major phases and psychological associations in orthognathic surgical protocols.

\section{Risk of passive agreement}

In adolescence, patients are often susceptible to following prescriptions without being personally involved in their treatment. This passiveness first risks taking the form of persecution at the time of surgery or following modifications resulting from surgery. Thus, regardless of the objective gravity of the deformity, it is primarily the discomfort that this deformity causes the patients in their daily life that must be investigated before committing to treatment.

However, this phenomenon is not limited to adolescence; Mrs. Paule (Figure 4) perceived the entire process of treatment as theft of her identity. Her initial request was for treatment to decrease loosening of her teeth and she accordingly found herself embarking on the course of orthognathic surgical treatment. She had learned to live with her 
prognathism: "It gave me something that made me stronger." Above all, she had no esthetic requests or functional discomfort. She was deeply upset by her medical process. "They told me I was sick when I was feeling fine. They turned me into a monster; I felt like I had to do something." The feedback from her relatives after treatment, who told her they noticed an improvement, was also disconcerting: "So, I was ugly before? I never perceived myself as such. I was me. And now I feel like I'm not anyone; I don't even know the woman I was before."

Risk of associating the deformity with one's previous failures

This can take the form of body dysmorphic disorder, of which patients are not aware during their initial request. We treat cases of body dysmorphic disorder already present in the initial request just after the request. This risk applies to older patients, who have sometimes already undergone orthodontic treatment during adolescence and to those whose initial request is esthetic and relates to tooth alignment. If a consideration of surgery requires a reevaluation of the patients' request (simply on an esthetic level: Do they want to change the lower part of their face? Are they bothered by the discrepancy in their jaw or only by the nonalignment of their teeth?). This adjustment can cause the disharmony to become the central focus of their previous difficulties. To provide evidence of cases in which treatment will definitely be dissatisfying and very costly psychologically, we introduce a clinical case.

Mr. Andre attended an orthognathic surgical consultation for the first time on the advice of his dentist. He was approximately 35 years old and after a period of social and professional stagnation, he had decided to take charge of his life, particularly on a medical level. His problem was essentially esthetic and preventive; he had no functional problems at the time. It is important
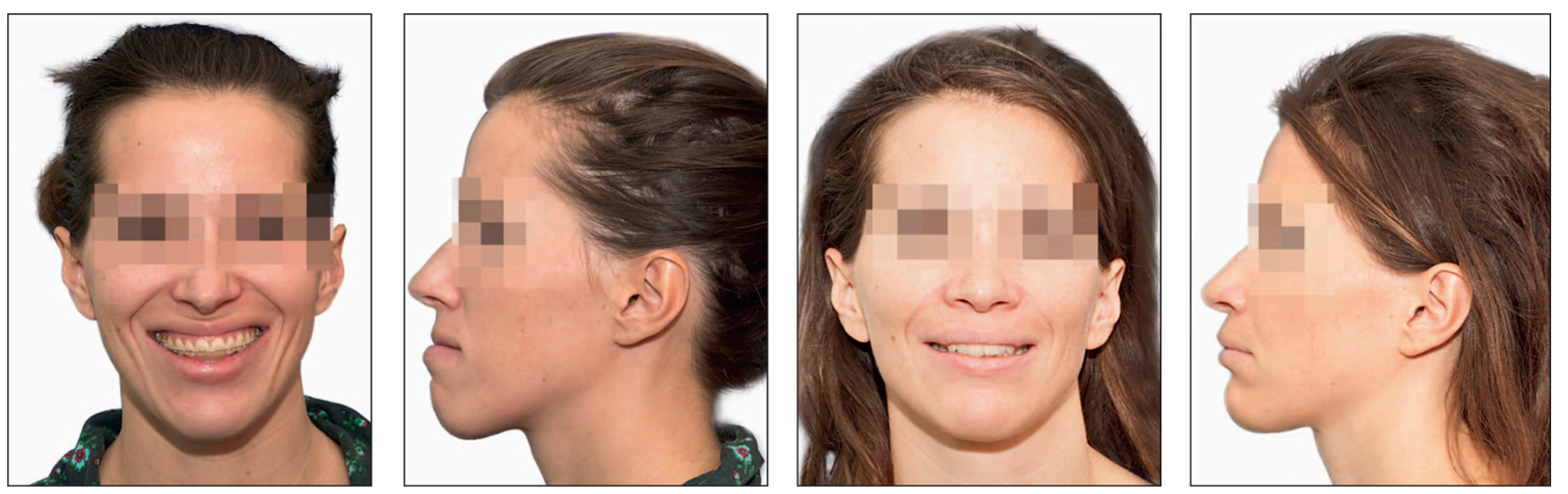

Figure 4

Mrs. Paule perceived her entire treatment as an identity violation. 
to note that his treatment process was based on his decision to regain control of his life. Orthognathic surgical treatment was part of this process, through which he wished to become what he had always wanted to be. I suggested he come in for an interview with us, to which he readily agreed. We then committed to a psychological follow-up, once per week, for him to start the necessary dental treatment before commencing orthognathic surgical treatment. He described his orthodontic journey as "chaotic" because of the interdisciplinary approach whereby communication passed "in all sorts of directions."

At the same time, he expressed a desire for orthodontic treatment because he was afraid that others considered him unapproachable; however, he was simply afraid to smile. He attributed this problem to the difficulty he experienced trying to overcome his fear of going on stage (in the entertainment world), all the while being afraid to smile and expose his teeth. He identified his bad teeth as the factor preventing him from succeeding in the entertainment world, like a brake in his relationship with others and a source of diminished self-confidence. $\mathrm{He}$ regretted not having started the orthognathic surgical process sooner, but it had been impossible at the time. "At first, it was because I was stupid and immature, and later because I did not have enough money. Now I want people to finally see me as I am; that is, as someone who is self-assured, approachable and open."

Mr. Andre finds himself stuck between two discourses, associated with his two expectations; that of maternal recognition in pursuit of conformity and of paternal recognition directed toward individuality. The dental issue specifically established in this dialectic was having a "very narrow" jaw. Perceiving himself as different meant he could not present himself as he truly was. In fact, it was the conflict between conformity and originality that marked his entire process, and by opting for originality, he discontinued the follow-up. This choice finally introduced a sense of continuity to his perceived identity: the link between these two discourses and these two images that allowed him to say "I." At the beginning of his treatment, Mr. Andre had not once performed the necessary dental care and chose not to begin the orthognathic surgical protocol. He received a professional promotion and stopped his psychological follow-ups. This does not mean that a patient cannot begin treatment at a later date, but by then the request would be related to medical problems driven by the need for a treatment process.

This case, which is no longer exceptional in terms of the problems we often encounter during preliminary interviews before commencing an orthognathic surgical protocol, really reflects the point we are trying to make. Some requests largely exceed the boundaries of ortho-surgery and cannot, through treatment, lead to a satisfying result. In order not to overlook inappropriate requests and to avoid passive agreement, it is necessary not to anticipate the request and to evaluate the request before even proposing to start treatment. Indeed, from the outset, some patients seek medical confirmation for 
inappropriate requests concerning, for example, their preoccupations with body dysmorphic disorder such as excessive daily focus on a daily basis to a particular body part invisible to the eye of a nonexpert and awaiting medical confirmation. This should immediately alert the practitioners and guide them in avoiding making any medical judgment (even if objectively justified). The practitioners should also direct the patients to receive a psychological consultation before proposing all other treatments.

This focus on the initial request will determine how the treatment progresses and takes place during the first multidisciplinary consultation in ortho-surgery when any risks that have previously been mentioned remain applicable. This is because the patients often expect to receive confirmation or valid information regarding treatment in this consultation.

\section{Stage 2: Preoperative orthodontics}

This stage is the ideal time to contemplate medium term psychological care for patients in whom one of the risks that we previously cited are perceived by the care team. The function of the follow-up system is to review the request with the patients to identify any underlying wishes, such as the desire to be recognized, to achieve an ideal, or to combat depression. Two types of pattern can be observed during this stage. For patients personally engaged in the treatment protocol and whose requests are adapted to the possibility of satisfaction, orthodontic treatment offers initial relief despite the pains that are associated with it. Undergoing treatment and having to wear braces causes others to consider the patients as different because they enter the category of "being in treatment." Making this choice produces the first therapeutic psychological effect. This is evident in the case of Mrs. Julia, who experienced absolutely no dysmorphia as a pathological outcome despite the significance of her discrepancy. However, from the moment her discrepancy was explained to her, she equated it to a sickness and had a tough time living with the situation (Figure 5). For patients who are more passively involved, this stage is decisive for evaluating their determination to pursue treatment. Indeed, it is not just the wearing of braces that results in previously unknown discomfort but also the preoperative alveolar decompensation that worsens the dysmorphia. The major risk at this stage is that the patients follow the treatment but does not express their sense of alienness toward themselves. This type of patient remains very reserved and compliant but presents a real risk of preoperative or postoperative decompensation.

Another clinical case highlights this pattern of behavior. Mr. Baptiste, aged 17, remained silent during his multidisciplinary consultations, to which he was accompanied by his father, and claimed that he was determined to follow the course of treatment when asked his opinion: "I have started and now I want to finish; I won't stay like this."

I meet him in a routine interview during a preoperative day visit 
approximately 1 month before surgery. These allow the analysis of, with the help of the patients' assistance, not only how the patients have prepared themselves for surgery but also what they fear but also what they expect, what they imagine, or what they have completely ignored. During this interview, Mr. Baptiste began to gradually express what he was feeling. He has no expectations of the surgery and he was involved in the treatment "because I was told that I had to do it although I do not really care." Over the course of the last few months before surgery, he admitted to feeling "strange": "I don't know. I no longer have the same head; my mouth was not doing this before. The surgery will surely return me to normal." By questioning this feeling of "strangeness," the patients evoked feelings of derealization. "At times I feel like I see myself as if I'm seeing double; I see myself obey, I see my body make movements, but I am not inside."
While talking to the patients' family, I learned that the young patient had in effect been "strange" for a number of months. He had consulted a psychiatrist who had prescribed him some antidepressants but his state seemed to be worsening week on week. The decision to postpone the surgery was evident and did not seem to affect the patients at all. This therefore posed the question of the removal of the braces and of psychiatric hospitalization. The period of adolescence is so delicate that conditions are prone to lead to psychosis.

This clinical insight exposes the risk of preoperative decompensation. Because the patients were as very reserved and compliant, it is conceivable that without psychological consultation the surgery would have continued as planned, thereby leading to postoperative psychological decompensation. The less "obvious" cases are often more prone to lead to such difficulties because they do not
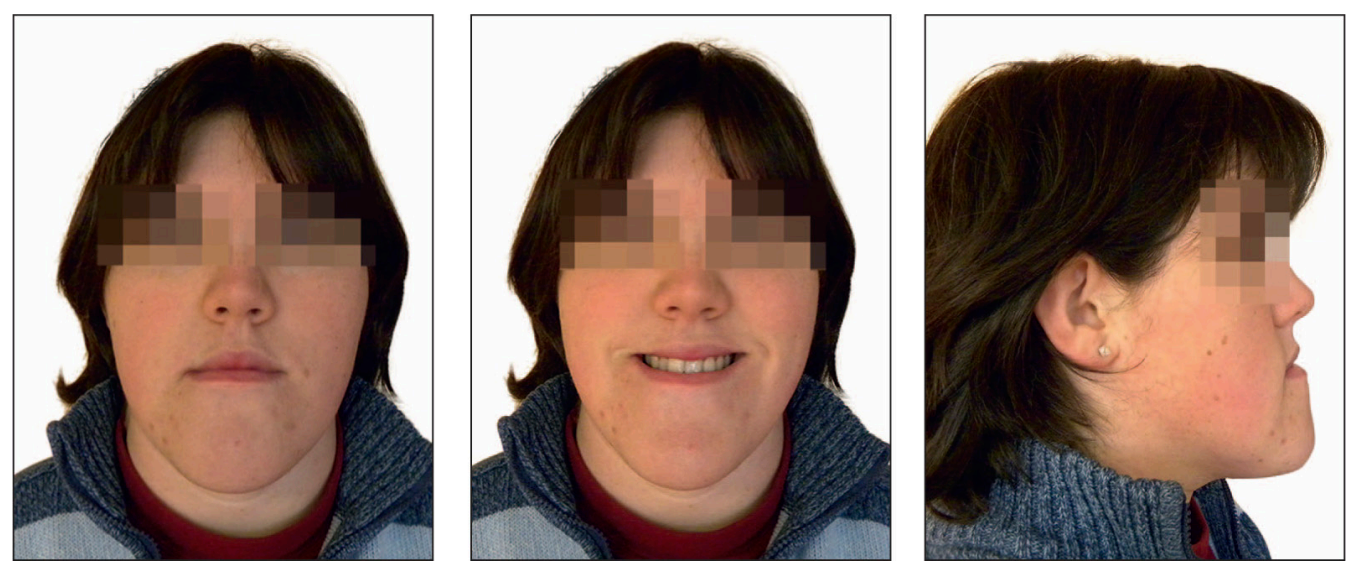

Figure 5

Mrs. Julia equated her dysmorphia with an illness in order to start treatment. 
necessarily draw the attention of the team. We therefore need to question the patients' request from as early as the first consultation before even giving a diagnosis or proposing treatment.

\section{Stage 3: Postoperativ e orthodontics}

In the month that follows surgery, patients often recount depressive episodes. "I really believed that I was never going to come out of this state. Even now I feel as if I haven't returned to normal. I get exhausted easily." "I really thought I was prepared but I didn't understand anything that was happening to me. I felt alone and misunderstood. I cried all the time and I did not want to see anyone. I no longer recognized myself." This difficult process is almost routine and can and must be prepared for during preoperative hospital visits, with care taken not to downplay the difficulties encountered by the patients. The role of image is central to depression. The "self" is no longer able to keep up with demands. Of a person's entire body image, the face is what appears as the first point of recognition. Any modification related to the face will undoubtedly lead to sensorimotor
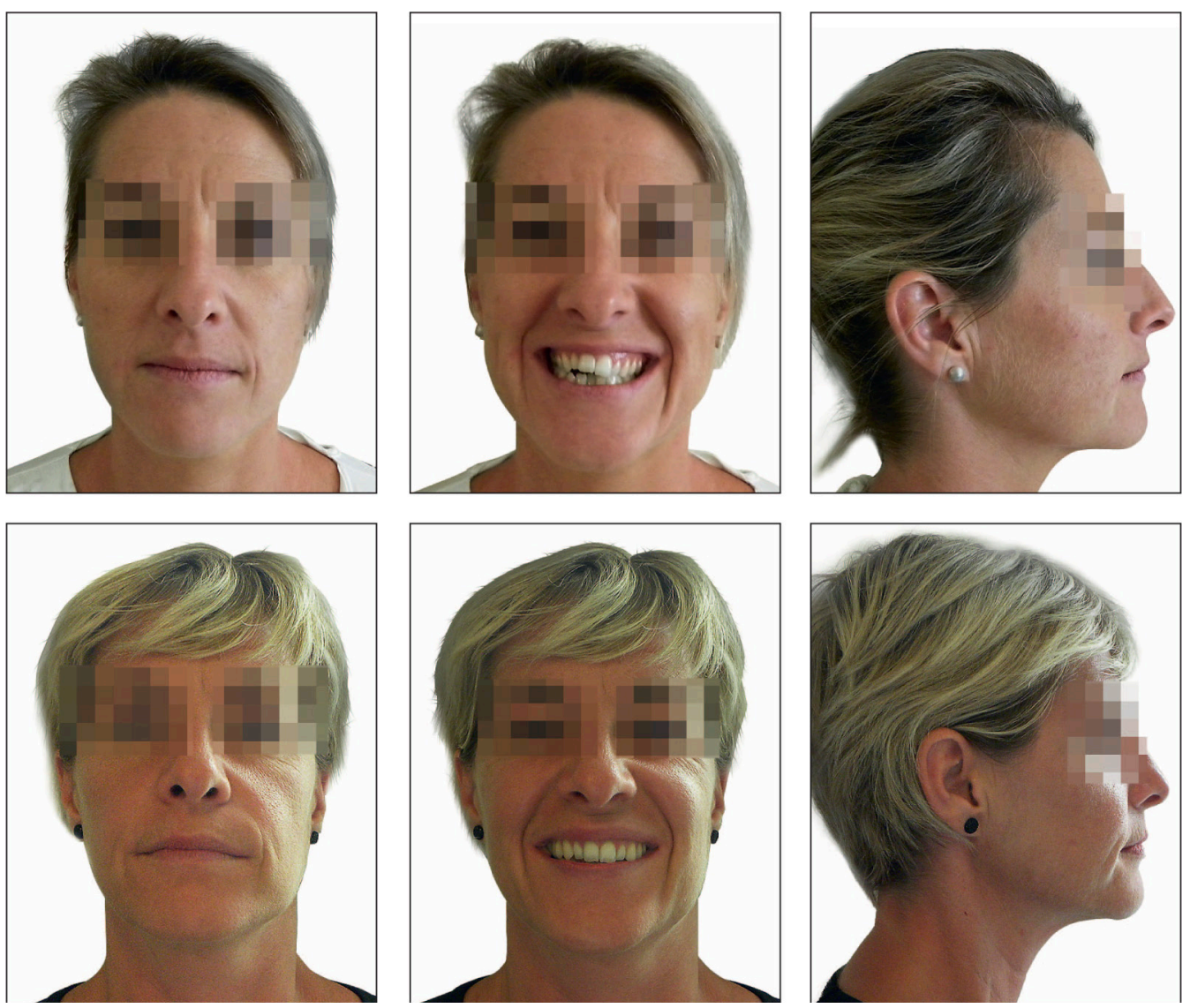

Figure 6

Mrs. Stéphanie changed her look after treatment. 
and psychological adjustments of one's relationship with oneself and others. The patients will handle the loss in such as the way that depressive feelings are cause for reorganization. Some traumatic elements linked to self-image can surface in this moral breakdown during the course of orthognathic surgical treatment. These elements can cause the patients to isolate themselves, thereby making it more difficult for them to call for help ${ }^{3}$. It is therefore vital that good relationships are maintained between the care team, the psychologists, and the patients at the end of treatment. This is of course evaluated depending on the needs of each party. The availability of the team will be the deciding factor in proceeding to the next step.

Stage 4: The conflict phase; appliance removal

This is the stage where the patients resume their activities of daily liv-

\section{DISCUSSION}

With any orthognathic surgical protocol, certain complications are prone to arise during the course of treatment. These complications are even desirable and they should not necessarily alarm the care team from the moment where listening and acceptance of the patients remain vital. The role of the psychologist in this process is threefold. They bring a specialist perspective to the intended benefits by analyzing the request, proposing a safe space to speak freely within which the patients can express their personal issues regarding ing "just as before." Although this is expected, it is worrisome for the patients. The braces are removed at this time, approximately 6 months after surgery, when the face has naturally settled into its final form and the treatment is complete. In this stage, we must remain vigilant of the expected changes to the patients' relationship with themselves and others and what is actually happening. Indeed, the treatment can be a lever for the patients to regain control of their life. We accordingly monitored the transformation of Mrs. Stéphanie's look (Figure 6), in which the roles of the environment and care team were key. The main risk at this stage is disappointment arising from unmet expectations. If the expectations were excessive, they should have been addressed before to ensure satisfaction at the end of the process. A match between the result of the treatment and what the patients originally expected is desirable.

the medical discourse, and developing a medium- to long-term follow-up system to assist with the patients' personal psychological adjustments during treatment.

The qualitative results that we have presented distinguish the necessary psychological adjustments from the psychological risks that remain subject to clinical impressions. To more closely support practitioners who undertake these challenges, a more complete study is needed. This study should deal specifically with quantitative and 
qualitative analyses of initial requests and of their evolutions during the course of treatment. This should allow the introduction of technical tools such as questionnaires and patient interviews (such as those used to address the patients during the initial consultation and preoperative consultation). These tools could more easily direct practitioners to propose psychological care to their patients.

\section{PROPOSAL FOR A RESEARCH PROTOCOL}

\section{Objectives and perspectives}

The main objective of the study will be to investigate the association between initial patient requests and the degree of patient satisfaction, while identifying certain profiles of request for which treatment may not work.

The secondary objectives will be to evaluate the evolution of the scoring for self-esteem, anxiety and depression, and quality of life in the three phases of research. More specifically, this will involve measuring the impact of psychological follow-up care on this evolution and describing the necessary psychological adjustments over the course of treatment.

Following this study, we intend to validate a questionnaire on initial requests, which will allow surgeons and orthodontists to refer patients for whom the orthognathic surgical protocol seems inappropriate or who are likely to pose a problem to a psychologist.

\section{Materials and methods}

\section{Population}

The study will be observational and prospective and the patients included will be followed-up longitudinally for
3 years (average time between initial consultation and 6 months postoperatively). The entire duration of the study will be 4 years: 1 year of inclusion and 3 years of participation (Figure 7). The patients will be registered for treatment with Professor Goudot's Maxillofacial Surgery Services at Pitié-Salpêtrière Hospital in Paris, France.

Inclusion criteria: Consenting patients over the age of 14 years who have received orthognathic surgical treatment for maxillomandibular deformities from the Maxillofacial Surgery Service at Pitié-Salpêtrière Hospital. If the child is a minor, written consent must be provided by the parents to show that they agree to their child participating in the research.

Exclusion Criteria: Patients with disharmony arising from post-traumatic or post-tumor aftereffects, syndromatic disharmony, endocrine disorders or growth disorders (such as temporomandibular ankylosis or condylar hyperplasia). Also excluded are patients with serious, life-threatening illnesses, no proficiency in the French language, and no social security coverage.

\section{Evaluation instruments}

For the past year, we have developed and tested a clinical 
questionnaire (Ortho-surgery Psychology Pitié-Salpêtrière [OPPS] questionnaire). This questionnaire was developed by Clément A, Guilyardi $H$, Dombrosky $M$, and Ney $M$ and analyzes changes in self-perception, psychosocial context, health, and request of the patients.

The preoperative questionnaire (Q1) comes in two versions: one for Phase 1 (Q1a) and the other for Phase 2 (Q1b). These two versions are similar. Included at the beginning is an agreement to participate in the research, which must be confirmed with an "I agree/refuse" response. The questionnaire uses a rating scale from 1 to 5 (1 indicating "total disagreement" and five indicating "complete agreement").

The postoperative questionnaire (Q2) reviews the first three elements by reintroducing them into a post-treatment context. A fourth part labeled "Results" evaluates patient satisfaction by asking respondents to reflect on the course of treatment as well as the changes that treatment has caused in the patients' daily lives. A score out of 45 calculated from nine items is used to measure the level of dissatisfaction. These self-administered questionnaires will be completed using three standardized scales:

\section{- Psychological interview after the first multidisciplinary consultation \\ - Self-administered Q1a, Rathus, HADS, and SF-36 scales \\ STAGE 1 \\ - Clinical observation matrix, proposal of psychological follow-up}

- Perioperative and postoperative psychological interviews

- Self-administered Q1b, Rathus, HADS, and SF-36 scales

STAGE 2

- Clinical observation matrix, proposal of psychological follow-up

STAGE 3

- Postoperative psychological interview

- Self-administered Q2, Rathus, HADS, and SF-36 scales

- Clinical observation matrix, proposal of psychological follow-up

Figure 7

Research protocol. 
- The Rathus Assertiveness Scale (Rathus Scale)20, composed of 30 statements describing situations in a patient's daily life. When responding, the patients must indicate to what degree these statements describe their behavior. The proposed scoring system is a six-point Likert scale. A score is obtained by totaling the scores of direct and reverse items.

- The Hospital Anxiety and Depression Scale (HADS) ${ }^{15}$ developed by Zigmond and Snaith (1983), which is intended to identify symptoms of depression and anxiety and to evaluate their severity by removing somatic symptoms likely to distort the evaluation. The French version has been validated by Lépine et al. (1985) and by Razavi et al. (1989). Only one version exists comprising 14 items (7 for depression and sev- en for anxiety). The scale is self-administered, quick to complete, and easy to use. Each item is answered on a four-point scale and scored 0-3 points. The overall score ranges from 0 to 42 (0-21 for each scale) and a higher score indicates a more severe symptomatology.

- The 36-item Short Form Health Survey (SF-36), which is a general, self-administered questionnaire on quality of life. SF-36 works on the assumption that everyone has health problems. The quality of life scale is comprised of physical functioning, general health perceptions, emotional role functioning and social role functioning. SF-36 evaluates eight health dimensions, which are each scored from 0 to 100 points. A score closer to 100 indicates a better quality of life.

\section{CONCLUSIONS}

This prospective longitudinal study is original in terms of its analysis of the psychological elements that underlie a patient's initial request rather than the mere establishment of psychological profiles. It is intended to help limit feelings of dissatisfaction in patients following orthognathic surgical treatment. This study also sets itself apart by allowing for an in-depth analysis by means of medium-term or long-term psychological follow-up that take place throughout treatment as a way of improving the patients' overall care.
The ultimate aim of the study is to analyze the effects of medical discourse on the development of a patient's request. This analysis could be used to provide practitioners with real tools that would help avoid anticipating requests and as such encourage exchanges that lead to both patient and practitioner satisfaction at the end of treatment.

Conflicts of interest: The authors declare that they have no conflicts of interest. 


\section{BIBLIOGRAPHY}

1. Battini J. Impact psychologique des interventions en chirurgie orthognatique: insatisfaction post-opératoire et personnalité dimensionnelle. Thèse Doct État. Tours: Univ François-Rabelais, 2013.

2. Bellucci CC, Kapp-Simon KA. Psychological considerations in orthognathic surgery. Clin Plast Surg 2007;34:e11-16.

3. Benkimoun F. Demande esthétique: aspects psychologiques. Rev Odonto Stomat 2011;40:261-279.

4. Bohne A, Keuthen NJ, Wilhelm S, Deckersbach T, Jenike MA. Prevalence of symptoms of body dysmorphic disorder and its correlates: a cross-cultural comparison. Psychosomatics 2002; 43:486-490.

5. Chen B, Zhang ZK, Wang X. Factors influencing postoperative satisfaction of orthognathic surgery patients. Int J Adult Orthodon Orthognat Surg 2002;17:217-222.

6. Cheng LH, Roles D, Telfer MR. Orthognathic Surgery: the patients' perspective. Br J Oral Maxillofac Surg 1998;36:261-263.

7. Cunningham SJ, Harrison SD, Feinman C, Hopper C. Body dysmorphic disorder involving the facial region: a report of 6 cases. J Oral Maxillofac Surg 2000;58:1180-1188.

8. Cunningham SJ, Gilthorpe MS, Hunt NP. Ares pre-treatment psychological characteristics influenced by pre-surgical orthodontics? Eur J Orthod 2001;23:751-758.

9. Espeland L, Hogevold HE, Stenvik A. A 3-year patient-centred follow-up of 516 consecutively treated orthognathic surgery patients. Eur J Orthod 2008;30:24-30.

10. Finlay PM, Atkinson JM, Moos KF. Orthognathic surgery: patient expectations; psychological profile and satisfaction with outcome. Br J Oral Maxillofac Surg 1995;33:9-14.

11. Garvill J, Garvill H, Kanhberg KE, Lundgren S. Psychological factors in orthognathic surgery. J Craniomaxillofac Surg 1992;20:28-33.

12. Kiyack HA, McNeill RW, West RA. The emotional impact of orthognathic surgery and conventional orthodontics. Am J Orthod 1985;88:224-234.

13. Kiyack HA, McNeill RW, West RA, Hohl T, Heaton PJ. Personality characteristics as predictors and sequelae of surgical and conventional orthodontics. Am J Orthod 1986;89:383-392.

14. Lacan, J. Le stade du miroir comme formateur de la fonction du Je. In Écrits pp 93-100. Paris: Seuil, 1966.

15. Langevin V, François M, Boini S, Riou A. Hopital Anxiety and Depression Scale. TC 134, Doc Medecin du Travail, nº 127, troisième trimestre 2011.

16. Lee S, McGrath C, Samman N. Impact of orthognathic surgery on quality of life. J Oral Maxillofac Surg 2008;66:1194-1199.

17. Merlini C, Placente M, Amelotti $C$, Antonioli M, Tadini C. Profil psychologique pré et post-opératoire des patients soumis à la chirurgie orthognathique. Rev Orthop Dento Faciale 1996;30:9-29.

18. Oland J, Jensen J, Melsen B, Elklit A. Are personality patterns and clinical syndromes associated with patients' motives and perceived outcome of orthognathic surgery? J Oral Maxillofac Surg 2010;68:3007-3014.

19. Olson RE, Laskin DM. Expectations of patients from orthognathic surgery. J Oral Surg 1980;38:283-285.

20. Rathus SA. A thirty item schedule for assessing assertive behavior. Behavior Therapy 4;298-406, 1973.

21. Vulink NCC, Rosenberg A, Plooij JM, Koole R, Berge J, Denys D. Body Dysmorphic disorder in maxillofacial outpatients presenting for orthognathic surgery. Int J Oral Maxillofac Surg 2008; 37:985-991. 